\title{
A Multi-cohort Study of the Prognostic Significance of Microsatellite Instability or Mismatch Repair Status after Recurrence of Resectable Gastric Cancer
}

\author{
Ji Yeong An, $\mathrm{PhD}{ }^{1}$ \\ Yoon Young Choi, PhD 2,3 \\ Jeeyun Lee, $\mathrm{PhD}^{4}$ \\ Woo Jin Hyung, $\mathrm{PhD}^{2}$ \\ Kyoung-Mee Kim, $\mathrm{PhD}^{5}$ \\ Sung Hoon Noh, PhD2 \\ Min-Gew Choi, $\mathrm{PhD}^{1}$ \\ Jae-Ho Cheong, $\mathrm{PhD} 23,6,7$
}

${ }^{1}$ Department of Surgery, Samsung Medical Center, Sungkyunkwan University School of Medicine, Seoul, ${ }^{2}$ Department of Surgery, Yonsei University Health System, Seoul,

${ }^{3}$ Yonsei Biomedical Research Institute, Yonsei University Health System, Seoul,

${ }^{4}$ Division of Hematology-Oncology,

Department of Medicine, ${ }^{5}$ Department of

Pathology and Translational Genomics,

Samsung Medical Center, Sungkyunkwan

University School of Medicine, Seoul,

${ }^{6}$ YUHS-KRIBB Medical Convergence

Research Institute, Seoul, ${ }^{7}$ Brain Korea

21 PLUS Project for Medical Science, Yonsei

University College of Medicine, Seoul, Korea

Correspondence: Jae-Ho Cheong, MD, PhD

Department of Surgery, Yonsei University

College of Medicine, 50 Yonsei-ro,

Seodaemun-gu, Seoul 03722, Korea

Tel: 82-2-2228-2094

Fax: 82-2-313-8289

E-mail: JHCHEONG @yuhs.ac

Received March 2, 2020

Accepted May 1, 2020

Published Online May 4, 2020

*Ji Yeong An and Yoon Young Choi contributed equally to this work.

\begin{abstract}
Purpose
High microsatellite instability (MSI) is related to good prognosis in gastric cancer. We aimed to identify the prognostic factors of patients with recurrent gastric cancer and investigate the role of MSI as a prognostic and predictive biomarker of survival after tumor recurrence.
\end{abstract}

\section{Materials and Methods}

This retrospective cohort study enrolled patients treated for stage II/III gastric cancer who developed tumor recurrence and in whom the MSI status or mismatch repair (MMR) status of the tumor was known. MSI status and the expression of MMR proteins were evaluated using polymerase chain reaction and immunohistochemical analysis, respectively.

\section{Results}

Of the 790 patients included, 64 (8.1\%) had high MSI status or MMR deficiency. The tumor-node-metastasis stage, type of recurrence, Lauren classification, chemotherapy after recurrence, and interval to recurrence were independently associated with survival after tumor recurrence. The MSI/MMR status and receiving adjuvant chemotherapy were not associated with survival after recurrence. In a subgroup analysis of patients with high MSI or MMR-deficient gastric cancer, those who did not receive adjuvant chemotherapy had better treatment response to chemotherapy after recurrence than those who received adjuvant chemotherapy.

\section{Conclusion}

Patients with high MSI/MMR-deficient gastric cancer should be spared from adjuvant chemotherapy after surgery, but aggressive chemotherapy after recurrence should be considered. Higher tumor-node-metastasis stage, Lauren classification, interval to recurrence, and type of recurrence are associated with survival after tumor recurrence and should thus be considered when establishing a treatment plan and designing clinical trials targeting recurrent gastric cancer.

\section{Key words}

Stomach neoplasms, Recurrence, Microsatellite instability, Prognosis, Biomarker

\section{Introduction}

Gastric cancer is among the leading causes of cancerrelated death worldwide [1,2]. Surgery and additional chemotherapy with and without radiation therapy are the standard treatment modalities for curative intent of locally advanced gastric cancer [3]. However, over $40 \%$ of patients treated with surgery and additional chemotherapy develop tumor recurrence that results in mortality [3,4]. Consequently, the prognostic factors of survival after recurrence (SAR) are clinically important in the treatment of gastric cancer. Nonetheless, only few studies have addressed this issue in 
gastric cancer $[4,5]$.

Recent studies on the biology of gastric cancer [6-8] have identified predictive biomarkers of prognosis and chemotherapy response [8-10]. Microsatellite instability (MSI) is one such biomarker. MSI-high (MSI-H) is known to be related to good prognosis and no benefit or even harmful effect from additional chemotherapy after surgery for stage II/ III gastric cancer $[4,11,12]$. These clinical characteristics imply that surgery alone would be an effective strategy for patients with MSI-H gastric cancer. However, some patients with MSI-H still experience tumor recurrence, and thus, clinicians hesitate while forgoing additional chemotherapy because of the fear that no chemotherapy may increase the risk of tumor recurrence [13]. It is unclear whether chemotherapy after recurrence yields a similar benefit between gastric cancer patients who did and did not receive postoperative chemotherapy before recurrence [5,14], particularly according to the MSI status. Thus, this study aimed to identify prognostic factors of patients with recurrent gastric cancer and investigate the role of the MSI status as a prognostic and predictive biomarker of SAR.

\section{Materials and Methods}

\section{Population}

Two large cohorts, namely the Y-cohort and S-cohort, were included in this study. The Y-cohort was based on the population of a previous study that reported the clinical implication of the MSI status for stage II/III gastric cancer [15], while the S-cohort was based on the population of previous studies on various biomarkers and molecular markers [7]. All patients underwent gastrectomy for curative intent and were pathologically confirmed to have stage II or III gastric cancer according to the 8th edition of the American Joint Committee on Cancer [16]. The additional chemotherapy and follow-up strategy were employed according to the guidelines $[17,18]$. We included patients with tumor recurrence and in whom the MSI or mismatch repair (MMR) status was known. Patients who received chemotherapy or radiation therapy before surgery and whose primary cancer was in the remnant stomach were excluded. Patients in whom the cancer was in the remnant stomach after initial gastrectomy but were treated via surgery with curative intent were also excluded.

\section{Clinicopathologic variables}

We retrospectively reviewed the clinicodemographic characteristics, including sex and age during initial gastric cancer diagnosis, presence of serosa invasion and lymph node metastasis, TNM stage, Lauren classification, location of tumor, MSI/MMR status, whether or not the patient received adjuvant chemotherapy, type of recurrence, inter- val between initial gastrectomy to tumor recurrence $(<1$ year, $\geq 1$ year and $<2$ years, $\geq 2$ years), and whether or not the patient received chemotherapy after recurrence. The type of recurrence was classified as locoregional, hematogenous, peritoneum, any combination, and ovarian metastasis only. Recurrence and survival were determined based on records from the hospital and the Korean National Statistical Office and through telephone surveys.

\section{Definition of MSI and MMR}

The MSI status was evaluated in the Y-cohort using polymerase chain reaction (PCR). DNA was extracted from paired normal tissue and tumor tissue that were formalinfixed, paraffin embedded, and amplified using PCR for two mononucleotide repeat markers (BAT25 and BAT26) and three dinucleotide markers (D5S346, D2S123, and D17S250) [19]. MSI-H was defined as an instability at two or more markers; otherwise, it was defined as microsatellite stable (MSS).

The expression of MMR proteins was evaluated in the S-cohort via immunohistochemical (IHC) analysis. Samples from tissue microarray blocks were used to prepare 3- to 4- $\mu \mathrm{m}$-thick sections for analysis, and monoclonal antibodies, including mouse anti-MLH1 (\#PA0610, Leica Biosystem, Buffalo Grove, IL), mouse anti-MSH2 (\#286M-16, Cell Marque, Rocklin, CA), mouse anti-MSH6 (\#610919, BD Transduction, San Jose, CA), and rabbit anti-PMS2 (\#288M-16, Cell Marque), were used for staining. Deficient MMR (dMMR) was defined as loss of any MMR protein expression in $\geq 80 \%$ of tumor cells while internal control nuclei (lymphocytes and stromal cells) are stained [20]. Two pathologists independently performed the evaluations.

\section{Statistical analysis}

Statistical analysis was performed using SPSS ver. 20.0 for Windows (IBM Corp., Armonk, NY) and R software ver. 3.4.3. Categorical variables are described as numbers and proportions and were compared using a chi-square test. Continuous variables are described as mean with standard deviation and were compared using an independent $t$ test. Patient prognosis was evaluated according to SAR, which was defined as the time from recurrence to death due to any cause. The SAR of each group was generated using KaplanMeier curves and compared using log-rank test. A Cox proportional hazard model was used with the hazard ratio (HR) and its 95\% confidence interval (CI). Institution was used as the adjustment variable for the overall analysis. Subgroup analysis was conducted with respect to each institution. The final multivariable model comprised variables that were statistically significant in the univariable analysis and were selected using likelihood forward methods. A two-sided p-value of $<0.05$ was considered statistically significant. 
Table 1. Clinicopathologic characteristics of the patients, initial gastric cancer stage, and treatments

\begin{tabular}{|c|c|}
\hline Characteristic & No. $(\%)(\mathrm{n}=790)$ \\
\hline Age, mean $\pm S D(y r)$ & $57.18 \pm 12.53$ \\
\hline \multicolumn{2}{|l|}{ Sex } \\
\hline Male & $521(65.9)$ \\
\hline Female & $269(34.1)$ \\
\hline \multicolumn{2}{|l|}{ Serosa invasion } \\
\hline Negative & $355(44.9)$ \\
\hline Positive & $435(55.1)$ \\
\hline \multicolumn{2}{|l|}{ LN metastasis } \\
\hline Negative & $64(8.1)$ \\
\hline Positive & $726(91.9)$ \\
\hline \multicolumn{2}{|l|}{ TNM stage } \\
\hline II & $188(23.8)$ \\
\hline III & $602(76.2)$ \\
\hline \multicolumn{2}{|l|}{ Lauren classification } \\
\hline Intestinal & $293(37.1)$ \\
\hline Diffuse/Mixed & $497(62.9)$ \\
\hline \multicolumn{2}{|l|}{ Tumor location } \\
\hline UB/Whole & $133(16.8)$ \\
\hline $\mathrm{MB} / \mathrm{LB}$ & $657(83.2)$ \\
\hline \multicolumn{2}{|l|}{ MSI/MMR } \\
\hline MSS/pMMR & $726(91.9)$ \\
\hline MSI-H/dMMR & $64(8.1)$ \\
\hline \multicolumn{2}{|l|}{ Adjuvant CTx ${ }^{a)}$} \\
\hline No & $214(27.3)$ \\
\hline Yes & $570(72.7)$ \\
\hline \multicolumn{2}{|c|}{ Interval to recurrence (yr) } \\
\hline$<1$ & $322(40.8)$ \\
\hline$\geq 1,<2$ & $237(30.0)$ \\
\hline$\geq 2$ & $231(29.2)$ \\
\hline \multicolumn{2}{|l|}{ Type of recurrence } \\
\hline Locoregional & $132(16.7)$ \\
\hline Hematogenous & $154(19.5)$ \\
\hline Peritoneum & $336(42.5)$ \\
\hline Any combination & $142(18.0)$ \\
\hline Krukenberg only & $26(3.3)$ \\
\hline \multicolumn{2}{|l|}{ CTx after recurrence ${ }^{a)}$} \\
\hline No & $265(35.1)$ \\
\hline Yes & $489(64.9)$ \\
\hline
\end{tabular}

LN, lymph node; UB, upperbody; MB, mid-body; LB, lower body; MSI, microsatellite instability; MMR, mismatch repair; MSS, microsatellite stable; pMMR, proficient mismatch repair; MSI-H, microsatellite instability high; dMMR, deficient mismatch repair;

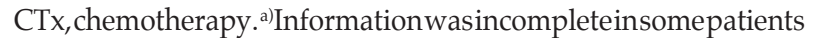
because of transfer to other hospital, loss of follow-up, and unclear medical records.

\section{Ethical statement}

This study was approved by the Institutional Review Board (approval number 4-2019-0244), and the need for informed consent was waived.

\section{Results}

1. Baseline characteristics of the patients, initial gastric cancer stage, and treatment

The overall cohort comprised 790 patients (439 and 351 patients from the Y-cohort and the S-cohort, respectively); of these, $65.9 \%$ were men. In total, 602 patients $(76.2 \%)$ were treated for stage III gastric cancer. With respect to Lauren histology, 293 (37.1\%) and 497 (62.9\%) patients had an intestinal and diffuse/mixed type, respectively. There were 64 patients with MSI-H/dMMR gastric cancer $(8.1 \%)$, and 570 patients $(72.7 \%)$ received adjuvant chemotherapy after gastrectomy. Over $70 \%$ of patients developed tumor recurrence within 2 years after initial surgery; the most common type was peritoneal recurrence $(42.5 \%)$. Furthermore, $64.9 \%$ of patients were administered chemotherapy after tumor recurrence (Table 1). The proportion of serosa-positive cases, MSI-H/dMMR cases, and cases treated with both adjuvant and post-recurrence chemotherapy; TNM stage; interval to recurrence; and type of recurrence were significantly different between the Y-cohort and S-cohort (S1 Table).

Consequently, institution was used as an adjustment variable in the following analysis. The type of recurrence was similar between MSI-H/dMMR and MSS/proficient MMR (pMMR) tumors (S2 Table).

\section{Factors related to prognosis after recurrence}

In the overall population, age (adjusted HR, $1.007 ; 95 \%$ CI, 1.011 to $1.013 ; \mathrm{p}=0.025$ ), serosa invasion (adjusted HR, 1.324; 95\% CI, 1.128 to 1.555; $\mathrm{p}=0.001$ ), TNM stage (adjusted HR, 1.288; 95\% CI, 1.086 to 1.527; $\mathrm{p}=0.004)$, Lauren classification (adjusted HR, 1.470; 95\% CI, 1.264 to 1.708; $p<0.001$ ), chemotherapy after recurrence (adjusted HR, 0.363; 95\% CI, 0.306 to $0.431 ; p<0.001$ ), interval from surgery to recurrence ( $p<$ $0.001)$, and type of recurrence $(p<0.001)$ were significantly associated with SAR (Table 2); in contrast, sex, presence of lymph node metastasis, location of tumor, and receiving adjuvant chemotherapy were not. The associations between clinicopathologic variables, except MSI/MMR status, and SAR were similar in both cohorts (S3 Table).

In the overall cohort, MSI-H/dMMR was not associated with SAR (adjusted HR, 1.155; 95\% CI, 0.885 to 1.506; $\mathrm{p}=0.290$ ) (Table 2, S4A Fig.). However, in the subgroup analysis by institutions, MSI-H was related to poor SAR in the Y-cohort (HR, 1.544; 95\% CI, 1.038 to 2.298; $\mathrm{p}=0.032$ ) (S3 Table, S4B Fig.), while there were no significant differences in SAR according to the MMR status in the S-cohort (HR, $0.950 ; 95 \% \mathrm{CI}, 0.667$ to $1.354 ; \mathrm{p}=0.777$ ) (S3 Table, S4C Fig.). In the institution-adjusted multivariable analysis, TNM stage, Lauren classification, interval to recurrence, type of recurrence, and chemotherapy after recurrence were independent factors related to SAR (Table 3). 
Table 2. Univariable Cox proportional hazard model for survival after recurrence

\begin{tabular}{|c|c|c|}
\hline & \multicolumn{2}{|c|}{ Overall cohort } \\
\hline & Adjusted HR ${ }^{a}(95 \%$ CI $)$ & p-value \\
\hline Age & 1.007 (1.001-1.013) & 0.025 \\
\hline Sex & & 0.362 \\
\hline Male & 1 & \\
\hline Female & $1.072(0.923-1.246)$ & \\
\hline Serosa invasion & & 0.001 \\
\hline Negative & 1 & \\
\hline Positive & $1.324(1.128-1.555)$ & \\
\hline LN metastasis & & 0.325 \\
\hline Negative & 1 & \\
\hline Positive & $1.141(0.878-1.482)$ & \\
\hline TNM stage & & 0.004 \\
\hline II & 1 & \\
\hline III & $1.288(1.086-1.527)$ & \\
\hline Lauren classification & & $<0.001$ \\
\hline Intestinal & 1 & \\
\hline Diffuse/Mixed & $1.470(1.264-1.708)$ & \\
\hline Tumor location & & 0.202 \\
\hline UB/Whole & 1 & \\
\hline $\mathrm{MB} / \mathrm{LB}$ & $1.135(0.934-1.378)$ & \\
\hline MSI/MMR & & 0.290 \\
\hline MSS/pMMR & 1 & \\
\hline MSI-H/dMMR & $1.155(0.885-1.506)$ & \\
\hline Adjuvant CTx & & 0.176 \\
\hline No & 1 & \\
\hline Yes & $0.894(0.760-1.052)$ & \\
\hline Interval to recurrence (yr) & & $<0.001$ \\
\hline$<1$ & 1 & \\
\hline$\geq 1,<2$ & $0.768(0.647-0.912)$ & 0.003 \\
\hline$\geq 2$ & $0.650(0.545-0.776)$ & $<0.001$ \\
\hline Type of recurrence & & $<0.001$ \\
\hline Locoregional & 1 & \\
\hline Hematogenous & $1.010(0.793-1.288)$ & 0.935 \\
\hline Peritoneum & $1.434(1.163-1.768)$ & 0.001 \\
\hline Any combination & $1.603(1.256-2.045)$ & $<0.001$ \\
\hline Krukenberg only & $0.614(0.393-0.961)$ & 0.033 \\
\hline CTx after recurrence & & $<0.001$ \\
\hline No & 1 & \\
\hline Yes & $0.363(0.306-0.431)$ & \\
\hline
\end{tabular}

HR, hazard ratio; CI, confidence interval; LN, lymph node; UB, upper body; MB, mid-body; LB, lower body; MSI, microsatellite instability; MMR, mismatch repair; MSS, microsatellite stable; pMMR, proficient mismatch repair; MSI-H, microsatellite instability high; dMMR,

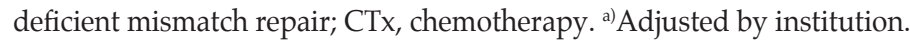

\section{Effect of chemotherapy according to MSI/MMR status}

Additional analyses were conducted to evaluate the effects of adjuvant chemotherapy and chemotherapy after recurrence in the overall cohort and according to the MSI/ MMR status. In the overall cohort, patients who received chemotherapy after recurrence had longer SAR regardless of whether or not they received adjuvant chemotherapy
( $\log$-rank $\mathrm{p}<0.001)$ (Table 4, Fig. 1A), and a similar finding was noted in both cohorts $(\log$-rank $\mathrm{p}<0.001$ and $\mathrm{p}<$ 0.001 in the Y-cohort and S-cohort, respectively) (S5A and S5B Fig.). A similar finding was observed in MSS/pMMR tumors of overall cohort and in both cohorts (Fig. 1B, S6A and S6B Fig.).

Among patients with MSI-H/dMMR tumors, those who 
Table 3. Multivariable Cox proportional hazard model for survival after recurrence in the overall cohort

\begin{tabular}{|c|c|c|}
\hline & Adjusted HR ${ }^{a)}(95 \%$ CI) & p-value \\
\hline TNM & & 0.049 \\
\hline II & 1 & \\
\hline III & 1.199 (1.001-1.435) & \\
\hline Lauren classification & & $<0.001$ \\
\hline Intestinal & 1 & \\
\hline Diffuse/Mixed & $1.515(1.294-1.775)$ & \\
\hline Interval to recurrence (yr) & & $<0.001$ \\
\hline$<1$ & 1 & \\
\hline$\geq 1,<2$ & $0.754(0.631-0.902)$ & 0.002 \\
\hline$\geq 2$ & $0.648(0.539-0.778)$ & $<0.001$ \\
\hline Type of recurrenc ${ }^{\text {b) }}$ & & $<0.001$ \\
\hline Locoregional/Hematogenous & 1 & \\
\hline Peritoneum/Combination & $1.326(1.085-1.620)$ & 0.006 \\
\hline Krukenberg only & $0.665(0.416-1.061)$ & 0.087 \\
\hline Chemotherapy after recurrence & & $<0.001$ \\
\hline No & 1 & \\
\hline Yes & $0.367(0.309-0.435)$ & \\
\hline
\end{tabular}

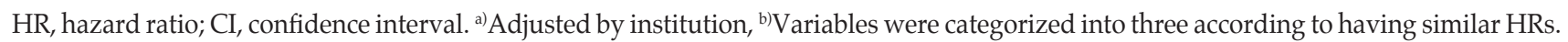

received chemotherapy only after recurrence had the longest SAR (Table 4), and the prognosis of patients who received adjuvant chemotherapy was similar regardless of whether they did or did not receive chemotherapy after recurrence (log-rank $\mathrm{p}=0.020$ ) (Fig. 1C). Similar findings were observed in the $\mathrm{Y}$-cohort (log-rank $\mathrm{p}=0.080$ ) and S-cohort (log-rank $\mathrm{p}=0.053$ ) (S7A and S7B Fig.).

\section{Discussion}

This study investigated the prognostic factors of patients with recurrent gastric cancer and the role of the MSI status as a prognostic and predictive biomarker of SAR. We found that among patients with MSI-H/dMMR recurrent gastric cancer, treatment response to chemotherapy after recurrence differed according to whether or not the patient received adjuvant chemotherapy. To the best of our knowledge, this is the first study to report such a result. This finding is clinically valuable because chemotherapy in the adjuvant setting would be detrimental to patients with stage II/ III MSI-H/dMMR gastric cancer when they develop tumor recurrence. Given that patients with MSI-H/dMMR stage II / III gastric cancer generally have favorable prognosis and that adjuvant/perioperative chemotherapy yields no benefit in reducing the risk of tumor recurrence $[4,11,12]$, these patients should be spared from adjuvant chemotherapy, with surgery alone being the most effective treatment strategy. This finding may be due to the acquired resistance from adjuvant chemotherapy and the negative effects of chemotherapy on patient immunity. The mechanism for this has been hypothesized as follows: (1) DNA-targeted cytotoxic chemotherapy increases treatment resistance in tumors lacking MMR activity, causing the selection of resistant MMRdeficient tumors, and this condition could increase genetic instability, heterogeneity, and selection of more invasive tumor cells [21]. Consequently, adjuvant chemotherapy for patients with MSI-H/dMMR gastric cancer could cause chemotherapy resistance without reducing the risk of tumor recurrence, and recurrence leads to poor prognosis. (2) MSI$\mathrm{H} / \mathrm{dMMR}$ tumors are related to enriched immune cells that may be responsible for the suppression of residual micrometastases after surgery [22,23], and chemotherapy may induce immune suppression [24]. In addition, chemotherapy may have a negative effect on immune surveillance, and the innate benefit from a hypermutated phenotype could be attenuated [12]. These negative effects of chemotherapy on the patient's immunity could lead to poor prognosis after tumor recurrence.

Our findings could lead to a clinical dilemma because not all patients with MSI-H/dMMR can be cured via surgery alone, and a tangible clinical benefit could be expected from adjuvant treatment in some patients with high-risk MSI-H/ dMMR gastric cancer $[13,25]$. Because patients with MSI-H/ dMMR tumors are possible candidates for immune-checkpoint inhibitor treatment [26,27], adjuvant immunotherapy would be a better strategy than conventional chemotherapy for this population. However, the superiority of adjuvant immunotherapy still needs to be verified. In addition, given that the prognosis of MSI-H/dMMR tumors could vary according to certain biomarkers [28,29], secondary biomarkers that can be used to guide adjuvant treatment for this spe- 
A

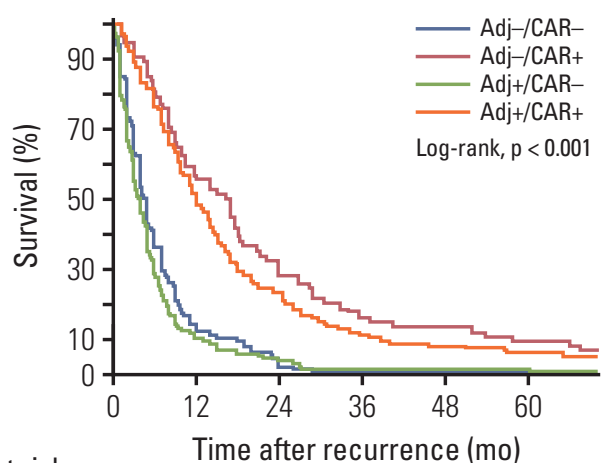

No. at risk

$\begin{array}{lllllll}\text { Adj-/CAR- } & 127 & 18 & 6 & 1 & 1 & 1\end{array}$

$\begin{array}{lllllll}\text { Adj-/CAR+ } & 74 & 42 & 22 & 12 & 10 & 7\end{array}$

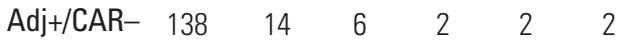

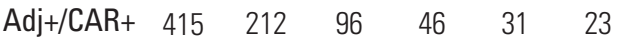

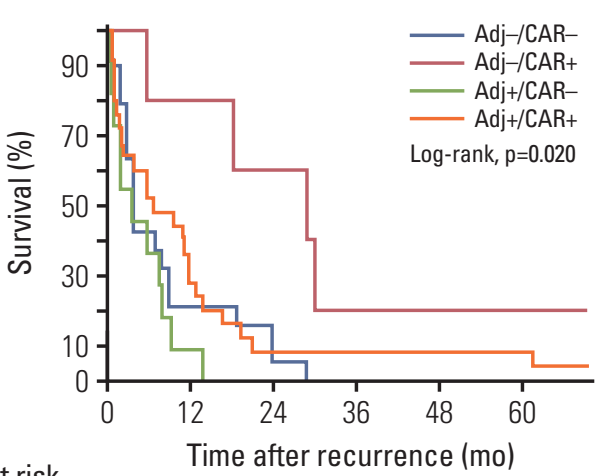

No. at risk

$\begin{array}{lrrrrrr}\text { Adj-/CAR- } & 19 & 4 & 3 & 0 & 0 & 0 \\ \text { Adj-/CAR+ } & 5 & 4 & 3 & 1 & 1 & 1 \\ \text { Adj+/CAR- } & 12 & 1 & 0 & 0 & 0 & 0 \\ \text { Adj+/CAR+ } & 25 & 9 & 2 & 2 & 2 & 2\end{array}$

\section{C}

B

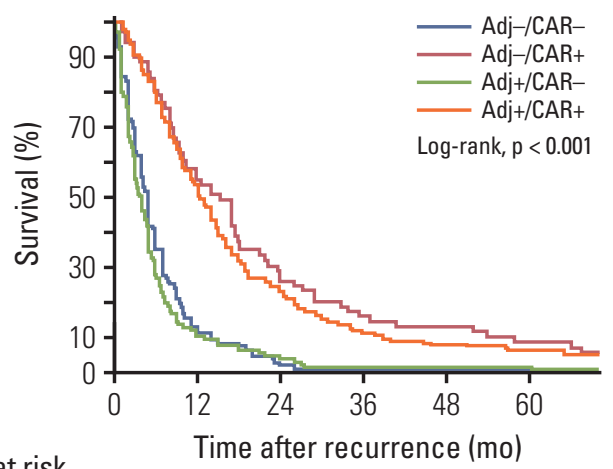

No. at risk

$\begin{array}{lllllll}\text { Adj-/CAR- } & 108 & 14 & 3 & 1 & 1 & 1\end{array}$

$\begin{array}{lllllll}\text { Adj-/CAR+ } & 69 & 38 & 19 & 11 & 9 & 6\end{array}$

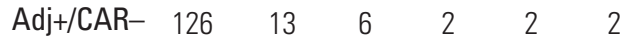

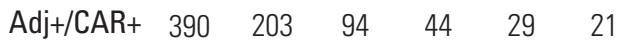

Fig. 1. Kaplan-Meier curves for survival after recurrence according to receiving adjuvant chemotherapy and chemotherapy after recurrence. (A) Regardless of the MSI/MMR status. (B) MSS / pMMR tumor. (C) MSI-H/dMMR tumors in the overall cohort. MSI, microsatellite instability; MRR, mismatch repair; MSS, microsatellite stable; pMMR, proficient MMR; MSI-H, MSI-high; dMMR, deficient MMR; n, number of patients; Adj, adjuvant chemotherapy; CAR, chemotherapy after recurrence.

cific type of gastric cancer should be investigated [13].

In this study, SAR was not statistically different according to the MSI/MMR status, but it differed according to the cohort. MSI-H was related to shorter SAR in the Y-cohort, while there was no difference in SAR between dMMR and pMMR patients in the S-cohort. This finding may be due to the following reasons: (1) the effect of chemotherapy might differ according to the MSI/MMR status, and the proportion of patients receiving adjuvant chemotherapy and chemotherapy after recurrence differed significantly between the cohorts in this study. (2) The Y-cohort was derived from a consecutive cohort enrolled between 2000 and 2010, while the S-cohort was derived from molecular or biomarker stud- ies conducted between 1995 and 2008. (3) The MSI/MMR status was assessed using different methods in the cohorts, i.e., PCR in the Y-cohort and IHC analysis in the S-cohort. In colorectal cancer, the association between the MSI/MMR status and prognosis after recurrence has been conflicting. A study on the molecular subtypes of colorectal cancer reported worse survival after relapse in those with MSI-H type [30], while a study of two randomized clinical trials reported longer SAR among patients with dMMR colon cancers [28] and attributed this to the difference in recurrence pattern according to the MMR status (regional vs. distant type). A similar recurrence pattern of MSI-H/dMMR and MSS/ pMMR tumors in this study resulted in a similar SAR, and 
Table 4. Association between treatment and survival after recurrence in the overall cohort and by MSI/MMR status

\begin{tabular}{|c|c|c|}
\hline & Adjusted HR ${ }^{\text {a) }}(95 \%$ CI) & p-value \\
\hline Overall & & $<0.001$ \\
\hline Adj-/CAR+ & 1 & \\
\hline $\mathrm{Adj}+/ \mathrm{CAR}+$ & $1.229(0.947-1.596)$ & 0.121 \\
\hline Adj-/CAR- & $2.820(2.062-3.856)$ & $<0.001$ \\
\hline Adj+/CAR- & $3.583(2.658-4.831)$ & $<0.001$ \\
\hline MSS/pMMR & & $<0.001$ \\
\hline Adj-/CAR+ & 1 & \\
\hline $\mathrm{Adj}+/ \mathrm{CAR}+$ & $1.133(0.867-1.481)$ & 0.359 \\
\hline Adj-/CAR- & $2.798(2.012-3.890)$ & $<0.001$ \\
\hline Adj+/CAR- & $3.356(2.464-4.570)$ & $<0.001$ \\
\hline MSI-H/dMMR & & $<0.001$ \\
\hline Adj-/CAR+ & 1 & \\
\hline Adj+/CAR+ & $5.360(1.472-19.517)$ & 0.011 \\
\hline Adj-/CAR- & $4.491(1.412-14.290)$ & 0.011 \\
\hline $\mathrm{Adj}+/ \mathrm{CAR}-$ & $12.052(3.065-47.391)$ & $<0.001$ \\
\hline
\end{tabular}

MSI, microsatellite instability; MMR, mismatch repair; HR, hazard ratio; CI, confidence interval; Adj, adjuvant chemotherapy; CAR, chemotherapy after recurrence; MSS, microsatellite stable; pMMR, proficient mismatch repair; MSI-H, microsatellite instability high; dMMR,

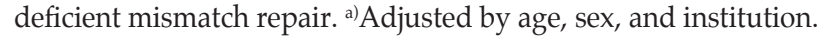

the effect of chemotherapy when administered before and after recurrence needs to be considered when evaluating the prognosis after recurrence according to the MSI/MMR status. Additional evidence is needed to determine whether the MSI/MMR status could be a useful biomarker even after tumor recurrence.

Given that initial tumor stage, Lauren classification, interval to recurrence, and type of recurrence were significantly related to SAR, these factors should be considered for patient stratification in clinical trials for recurrent gastric cancer. More advanced initial tumor stage may be related to more subclinical metastases and aggressive biologic behaviors that result in more progressive recurrence [14]. The diffuse histology of gastric cancer is related to cancer stemness and being refractory to chemotherapy $[6,8]$, and this could lead to shorter SAR. Tumors that recur at a longer interval from initial treatment may tend to behave in a more indolent manner after recurrence. Peritoneal and combination recurrence would be less responsive to oral or intravenous chemotherapy and may yield a higher tumor burden that could cause shorter SAR.

This study enrolled patients from the two largest gastric cancer-specialized centers in Korea. Considering the low prevalence of MSI-H/dMMR gastric cancer with its favorable prognosis, this cohort may be the largest to date. In addition, this study is the first to evaluate the effects of both chemotherapy before and after recurrence according to SAR biomarkers of gastric cancer. However, there are also possible limitations of this study that need to be addressed. The effects of chemotherapy after recurrence were likely overestimated as some patients died before chemotherapy could be implemented after tumor recurrence. Moreover, the retrospective design of this study and the different chemotherapy regimens used for adjuvant chemotherapy and chemotherapy after recurrence made it difficult to conduct more subgroup analysis for the effect of chemotherapies before and after recurrence by MSI/MMR status, and it might be another limitation. Despite these limitations, our results provide clinical insight into the behavior of MSI-H/dMMR gastric cancer in the setting of standard of care and provide instrumental information for deciding on the appropriate treatment strategy for recurrent gastric cancer.

In conclusion, chemotherapy only after recurrence yields high SAR in gastric cancer patients with MSI-H/dMMR. This shows that in MSI gastric cancer, only post-recurrence chemotherapy and not adjuvant chemotherapy is beneficial. Furthermore, TNM stage, Lauren classification, interval to recurrence, and type of recurrence are associated with SAR and should thus be considered when creating the treatment plan and designing clinical trials targeting recurrent gastric cancer.

\section{Electronic Supplementary Material}

Supplementary materials are available at Cancer Research and Treatment website (https:// www.e-crt.org).

\section{Conflict of Interest}

Conflict of interest relevant to this article was not reported. 


\section{References}

1. Bray F, Ferlay J, Soerjomataram I, Siegel RL, Torre LA, Jemal A. Global cancer statistics 2018: GLOBOCAN estimates of incidence and mortality worldwide for 36 cancers in 185 countries. CA Cancer J Clin. 2018;68:394-424.

2. Jung KW, Won YJ, Kong HJ, Lee ES. Cancer statistics in Korea: incidence, mortality, survival, and prevalence in 2016. Cancer Res Treat. 2019;51:417-30.

3. Noh SH, Park SR, Yang HK, Chung HC, Chung IJ, Kim SW, et al. Adjuvant capecitabine plus oxaliplatin for gastric cancer after D2 gastrectomy (CLASSIC): 5-year follow-up of an open-label, randomised phase 3 trial. Lancet Oncol. 2014;15: 1389-96.

4. Pietrantonio F, Miceli R, Raimondi A, Kim YW, Kang WK, Langley RE, et al. Individual patient data meta-analysis of the value of microsatellite instability as a biomarker in gastric cancer. J Clin Oncol. 2019;37:3392-400.

5. Ito S, Ohashi Y, Sasako M. Survival after recurrence in patients with gastric cancer who receive S-1 adjuvant chemotherapy: exploratory analysis of the ACTS-GC trial. BMC Cancer. 2018;18:449.

6. Cancer Genome Atlas Research Network. Comprehensive molecular characterization of gastric adenocarcinoma. Nature. 2014;513:202-9.

7. Cristescu R, Lee J, Nebozhyn M, Kim KM, Ting JC, Wong SS, et al. Molecular analysis of gastric cancer identifies subtypes associated with distinct clinical outcomes. Nat Med. 2015; 21:449-56.

8. Cheong JH, Yang HK, Kim H, Kim WH, Kim YW, Kook MC, et al. Predictive test for chemotherapy response in resectable gastric cancer: a multi-cohort, retrospective analysis. Lancet Oncol. 2018;19:629-38.

9. Yong WP, Rha SY, Tan IB, Choo SP, Syn NL, Koh V, et al. Real-time tumor gene expression profiling to direct gastric

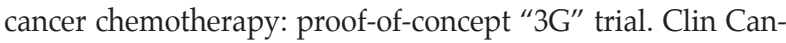
cer Res. 2018;24:5272-81.

10. Roh CK, Choi YY, Choi S, Seo WJ, Cho M, Jang E, et al. Single patient classifier assay, microsatellite instability, and Epstein-Barr virus status predict clinical outcomes in stage II / III gastric cancer: results from CLASSIC trial. Yonsei Med J. 2019;60:132-9.

11. Choi YY, Kim H, Shin SJ, Kim HY, Lee J, Yang HK, et al. Microsatellite instability and programmed cell death-ligand 1 expression in stage II/ III gastric cancer: post hoc analysis of the CLASSIC randomized controlled study. Ann Surg. 2019;270:309-16.

12. Smyth EC, Wotherspoon A, Peckitt C, Gonzalez D, HulkkiWilson S, Eltahir Z, et al. Mismatch repair deficiency, microsatellite instability, and survival: an exploratory analysis of the Medical Research Council Adjuvant Gastric Infusional Chemotherapy (MAGIC) trial. JAMA Oncol. 2017;3:1197-203.

13. Choi YY, Cheong JH. Comment on "To treat, or not to treat, that is the question: biomarker-guided adjuvant chemotherapy for stage II and III gastric cancer". Ann Surg. 2019;270: e40-1.

14. O'Connell MJ, Campbell ME, Goldberg RM, Grothey A, Seitz JF, Benedetti JK, et al. Survival following recurrence in stage
II and III colon cancer: findings from the ACCENT data set. J Clin Oncol. 2008;26:2336-41.

15. Kim SY, Choi YY, An JY, Shin HB, Jo A, Choi H, et al. The benefit of microsatellite instability is attenuated by chemotherapy in stage II and stage III gastric cancer: results from a large cohort with subgroup analyses. Int J Cancer. 2015;137:819-25.

16. Amin MB, Edge SB, Greene FL, Byrd DR, Brookland RK, Washington MK, et al. AJCC cancer staging manual. 8th ed. New York: Springer; 2017.

17. Guideline Committee of the Korean Gastric Cancer Association (KGCA), Development Working Group and Review Panel. Korean practice guideline for gastric cancer 2018: an evidence-based, multi-disciplinary approach. J Gastric Cancer. 2019;19:1-48.

18. Japanese Gastric Cancer Association. Japanese gastric cancer treatment guidelines 2014 (ver. 4). Gastric Cancer. 2017;20:119.

19. Boland CR, Thibodeau SN, Hamilton SR, Sidransky D, Eshleman JR, Burt RW, et al. A National Cancer Institute Workshop on Microsatellite Instability for cancer detection and familial predisposition: development of international criteria for the determination of microsatellite instability in colorectal cancer. Cancer Res. 1998;58:5248-57.

20. Cho J, Kang SY, Kim KM. MMR protein immunohistochemistry and microsatellite instability in gastric cancers. Pathology. 2019;51:110-3.

21. Meyers M, Wagner MW, Hwang HS, Kinsella TJ, Boothman DA. Role of the hMLH1 DNA mismatch repair protein in fluoropyrimidine-mediated cell death and cell cycle responses. Cancer Res. 2001;61:5193-201.

22. Grogg KL, Lohse CM, Pankratz VS, Halling KC, Smyrk TC. Lymphocyte-rich gastric cancer: associations with EpsteinBarr virus, microsatellite instability, histology, and survival. Mod Pathol. 2003;16:641-51.

23. Chiaravalli AM, Feltri M, Bertolini V, Bagnoli E, Furlan D, Cerutti R, et al. Intratumour T cells, their activation status and survival in gastric carcinomas characterised for microsatellite instability and Epstein-Barr virus infection. Virchows Arch. 2006:448:344-53.

24. Onyema OO, Decoster L, Njemini R, Forti LN, Bautmans I, De Waele $\mathrm{M}$, et al. Chemotherapy-induced changes and immunosenescence of CD8+ T-cells in patients with breast cancer. Anticancer Res. 2015;35:1481-9.

25. Mondaca S, Yoon SS, Strong VE, Ku GY, Ilson DH, Greally M, et al. Comment on "Microsatellite instability as a predictive biomarker for adjuvant chemotherapy in gastric cancer": are we there yet? Ann Surg. 2019;270:e39-40.

26. First tissue-agnostic drug approval issued. Cancer Discov. 2017;7:656.

27. Le DT, Uram JN, Wang H, Bartlett BR, Kemberling H, Eyring $\mathrm{AD}$, et al. PD-1 blockade in tumors with mismatch-repair deficiency. N Engl J Med. 2015;372:2509-20.

28. Sinicrope FA, Shi Q, Allegra CJ, Smyrk TC, Thibodeau SN, Goldberg RM, et al. Association of DNA mismatch repair and mutations in BRAF and KRAS with survival after recurrence in stage III colon cancers: a secondary analysis of 2 rand- 
omized clinical trials. JAMA Oncol. 2017;3:472-80.

29. Cho J, Lee J, Bang H, Kim ST, Park SH, An JY, et al. Programmed cell death-ligand 1 expression predicts survival in patients with gastric carcinoma with microsatellite instability.
Oncotarget. 2017;8:13320-8

30. Guinney J, Dienstmann R, Wang X, de Reynies A, Schlicker A, Soneson C, et al. The consensus molecular subtypes of colorectal cancer. Nat Med. 2015;21:1350-6. 